\title{
Characteristics of coronary artery disease in chronic kidney disease
}

\author{
Hideki Fujii $^{1}$ (D) Keiji Kono ${ }^{1} \cdot$ Shinichi Nishi ${ }^{1}$
}

Received: 1 December 2018 / Accepted: 7 February 2019 / Published online: 4 March 2019

(c) The Author(s) 2019

\begin{abstract}
Patients with chronic kidney disease (CKD) commonly experience cardiovascular disease (CVD), and a major cause of death in these patients is CVD. Therefore, the prevention of CVD progression is very crucial in patients with CKD. Recently, this relationship between CKD and CVD has increasingly been examined, and a concept termed "cardiorenal syndrome" has been advocated. Coronary artery disease (CAD) and myocardial injury are crucial factors that contribute to the occurrence of CVD. The initial step CAD is endothelial dysfunction that can be detected as a decrease in the coronary flow reserve (CFR). The previous studies have reported that decreased CFR is significantly correlated to coronary events and mortality. Furthermore, CFR reduces with a decline in the kidney function. Another important presentation of CAD is coronary artery calcification. Vascular calcification is a crucial pathophysiological state, particularly in patients with CKD, and it affects the stability of coronary atherosclerotic plaque. In CKD, not only the traditional risk factors but also CKD-related nontraditional risk factors play key roles in CVD progression. Therefore, the mechanisms responsible for CVD progression are very complex; however, their clarification is crucial to improve the prognosis in patients with CKD.
\end{abstract}

Keywords Cardiovascular disease $\cdot$ Coronary artery disease $\cdot$ Chronic kidney disease

\section{Introduction}

Chronic kidney disease (CKD) has recently been gaining attention, because many studies have demonstrated a strong correlation of CKD with mortality. Cardiovascular disease (CVD) is a well-known major cause of death in patients with CKD. Therefore, understanding the association between CKD and CVD is crucial. To this end, the concepts of "cardiorenal association" and "cardiorenal syndrome" have been advocated [1], and a large number of clinical and experimental studies have attempted to elucidate the detailed mechanisms. Furthermore, CKD is considered to be a crucial risk factor for CVD even in the field of cardiology [2].

Along with the classical risk factors such as hypertension, smoking, hyperlipidemia, diabetes mellitus, and aging,

This article was presented as the Clinical Scientist Award memorial lecture at the 61th annual meeting of the Japanese Society of Nephrology, held at Niigata, Japan in 2018.

Hideki Fujii

fhideki@med.kobe-u.ac.jp

1 Division of Nephrology and Kidney Center, Kobe University Graduate School of Medicine, 7-5-2, Kusunoki-cho,

Chuo-ku, Kobe, Hyogo 650-0017, Japan
CKD-specific non-classical risk factors contribute to CVD progression in CKD. CKD-specific non-classical risk factors include inflammation, anemia, volume overload, oxidative stress, renin-angiotensin system, sympathetic nerve system, uremic toxins, and chronic kidney disease-mineral bone disorder (CKD-MBD). As mentioned above, many studies have assessed the cardiorenal association; however, the detailed pathophysiological mechanisms remain unknown.

One of the crucial pathophysiological manifestations of CVD in CKD is coronary artery disease (CAD). The CAD prevalence and the number of severe coronary artery stenoses increase as kidney function deteriorates [3, 4]. However, several aspects regarding this subject including the pathophysiological mechanisms remain unclear. In the present review, we summarize the characteristics and possible pathophysiological mechanisms of CAD in CKD based on the present clinical and experimental evidences (Fig. 1). 
Fig. 1 Putative mechanisms of CAD in CKD. CAD coronary artery disease, $C K D$ chronic kidney disease, $C F R$ coronary flow reserve, $A C S$ acute coronary syndrome

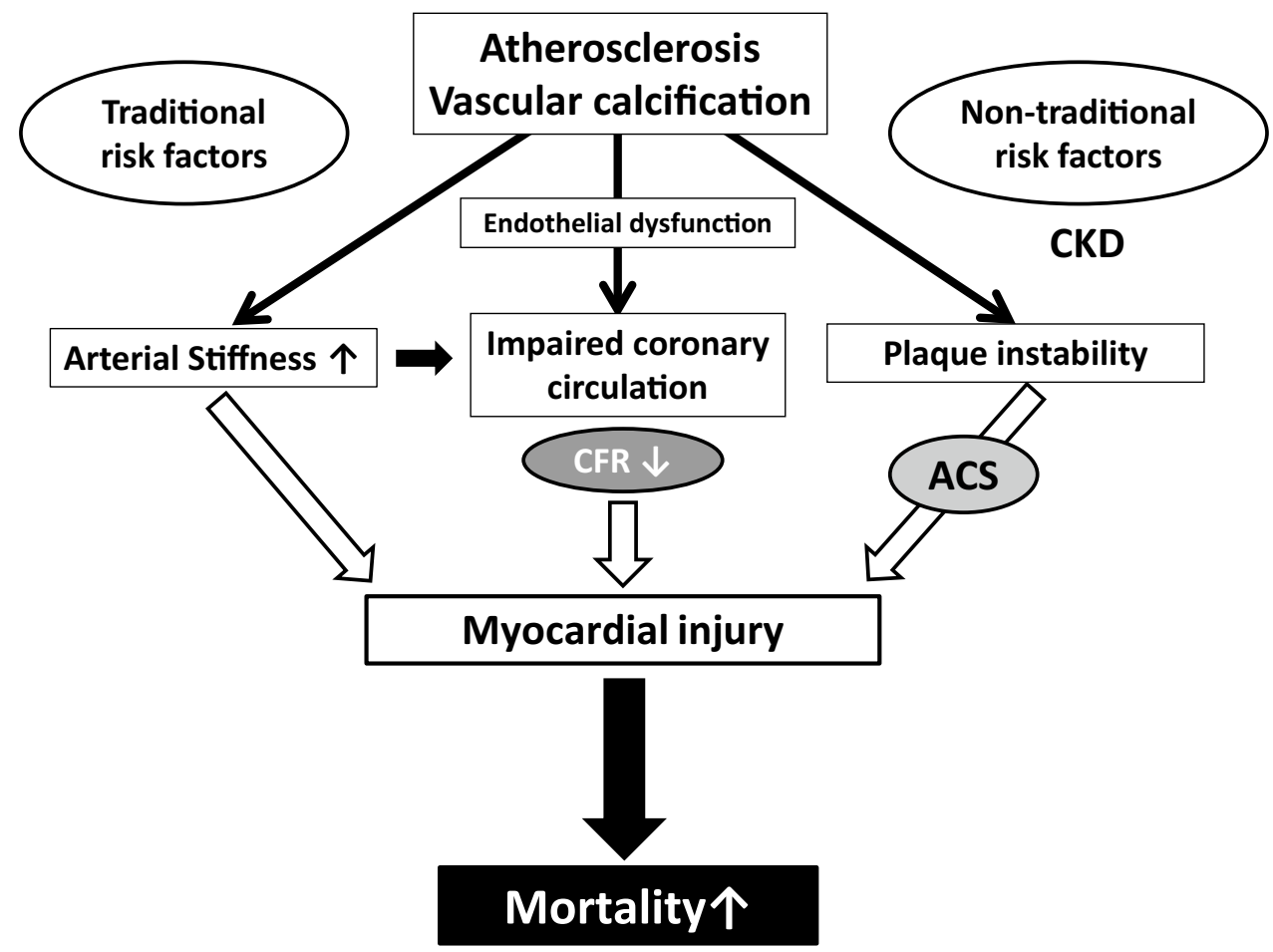

\section{Prevalence and clinical characteristics of CAD in CKD}

A previous study conducted in the United States has reported that the averaged estimated glomerular filtration rate (eGFR) in 14,527 patients with acute myocardial infarction (AMI) was $70 \pm 21 \mathrm{~mL} / \mathrm{min} / 1.73 \mathrm{~m}^{2}$, and $33.6 \%$ of these patients had CKD [5]. A Canadian study using a large population cohort demonstrated significantly higher incident AMI rates in patients with CKD than in those with diabetes [6]. Data of Japanese patients undergoing hemodialysis showed that the cause of death was AMI in $3.0 \%$ of these patients [7]. Moreover, based on the data of patients with non-dialyzed CKD and those undergoing hemodialysis in the United States, although the AMI prevalence increased with decrease in the eGFR, it decreased in patients undergoing hemodialysis [8, 9].

Several studies have evaluated the presence of CAD in asymptomatic patients new to hemodialysis $[10,11]$. These data demonstrated that approximately $50 \%$ of these patients already had CAD without any clinical symptoms. However, these studies were performed during the first decades of the 2000s and a recent study has reported that the CAD prevalence has decreased among these patients in recent years [12].

The diagnosis of CAD in patients with CKD is very challenging, because these patients do not demonstrate the typical clinical symptoms of CAD and do not show the typical changes observed in CAD on electrocardiogram (ECG), such as ST-T change and abnormal Q wave. An interesting study has compared the clinical symptoms of patients undergoing hemodialysis with those not undergoing hemodialysis [13]. The percentage of those with chest discomfort, those with ST-T changes, and those with an AMI diagnosis at the emergency department visits was significantly lower, and the percentage of those with pulmonary edema, cardiac arrest, and death during hospitalization was significantly higher in the hemodialysis group than in the non-hemodialysis group. Furthermore, patients with AMI who had lower kidney function had poor prognosis [5]. Thus, the presence of CKD is a crucial issue for patients with CAD.

\section{Endothelial dysfunction of coronary artery}

Endothelial dysfunction is the first step of atherosclerosis, and albuminuria is believed to reflect endothelial dysfunction. Endothelial dysfunction evaluated using acetylcholinestimulated forearm blood flow was significantly associated with CVD in patients with hypertension [14]. Moreover, not only decreased kidney function but also albuminuria is reportedly associated with an increased CVD risk [15, 16].

Impaired blood flow in the small intramural resistance vessels or in the coronary capillary system that cannot be visualized using coronary angiography results in decreased coronary microcirculation [17]. Coronary blood flow 
normally increases automatically from the resting level to the peak level in response to increases in the myocardial oxygen demand [18]. Such a change in the coronary blood flow is regarded the coronary flow reserve (CFR). Several studies have demonstrated that CFR is significantly associated with kidney function [19-21]. Several our clinical studies assessed CFR using transthoracic Doppler echocardiography (Fig. 2) [20, 22-24], a non-invasive and safe method that does not involve the risk of radiation exposure. Our data showed that CFR was significantly associated with the estimated glomerular filtration rate (eGFR) in hypertensive patients without a significant coronary artery stenosis (Fig. 3a) and that both CFR and eGFR were significantly associated with asymmetric dimethylarginine (ADMA), an endogenous competitive inhibitor of nitric oxide (NO) synthase (Fig. 3b, c) [20]. Therefore, decreased local NO production following an increase in ADMA may lead to impaired microcirculation in the kidneys and heart, particularly in CKD. In fact, the previous studies have reported that decreased CFR was related to mortality in patients with CKD not undergoing hemodialysis as well as in those undergoing hemodialysis [25, 26].

\section{CAC in CKD}

There are several risk factors for coronary artery calcification (CAC): age, diabetes mellitus, and CKD are believed to be the most important of these $[27,28]$. With population aging, the CAC prevalence is increasing and male sex has a greater impact on CAC [29]. Furthermore, racial differences are also observed with respect to CAC progression, with Asians reported to have a lower prevalence than in the Western countries, including United States [30]. The CAC prevalence is much lower in Japan than in the other countries. Decreased kidney function is an important risk factor of CAC, and it has been reported that the prevalence of severe CAC increases with worsening of kidney function [31]. At the initiation of hemodialysis therapy, $81 \%$ of the study subjects in our earlier study already had CAC; $43.8 \%$ had mild-to-moderate CAC, and $37.5 \%$ had severe $\mathrm{CAC}$ [32]. The CAD prevalence is higher in patients with $\mathrm{CAC}$ and the prevalence is related to CAC severity [33]. This finding is also supported by the results of several studies conducted on only patients with CKD [31, 34].

Vascular calcification is commonly observed in CKD, because, in addition to several classical risk factors, patients with CKD also have certain unconventional risk
A

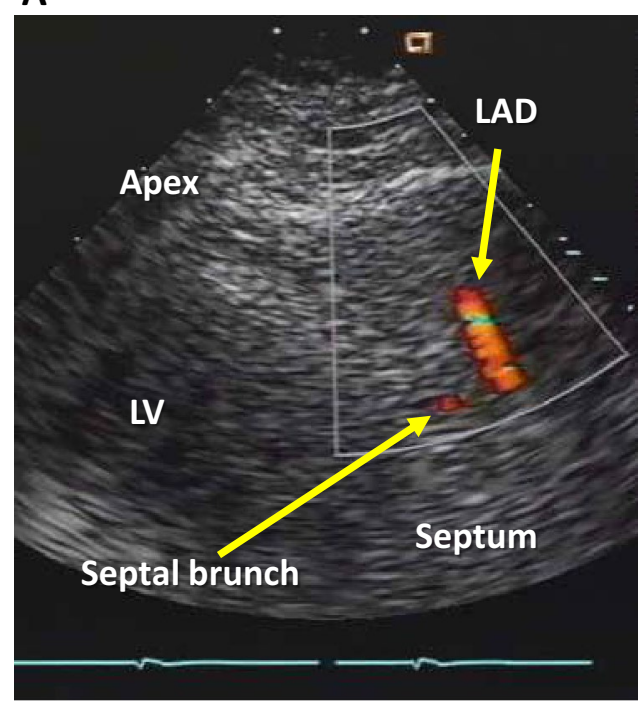

B

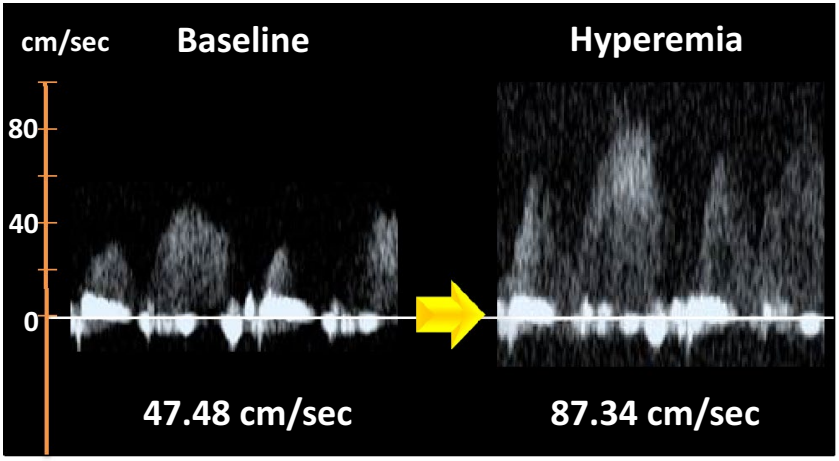

CFR 1.77 (normal: > 3.0)

\section{CFR=hyperemic MDV/basal MDV}

Fig. 2 Measurement of CFR. a Visualization of the coronary artery using transthoracic echocardiography. b Coronary flow velocity at baseline and during hyperemia in patients with abnormal CFR. a Imaging of LAD using transthoracic echocardiography. First, the MDV at baseline is measured; thereafter, the MDV during a hypere- mic condition induced by ATP administration ( $140 \mu \mathrm{g} / \mathrm{kg} / \mathrm{min} \mathrm{IV)} \mathrm{for}$ $3-5 \min \mathbf{b}$ is measured. CFR is calculated as the ratio of hyperemic MDV to basal MDV. $C F R$ coronary flow reserve, $L A D$ left anterior descending artery, $M D V$ mean diastolic velocity 


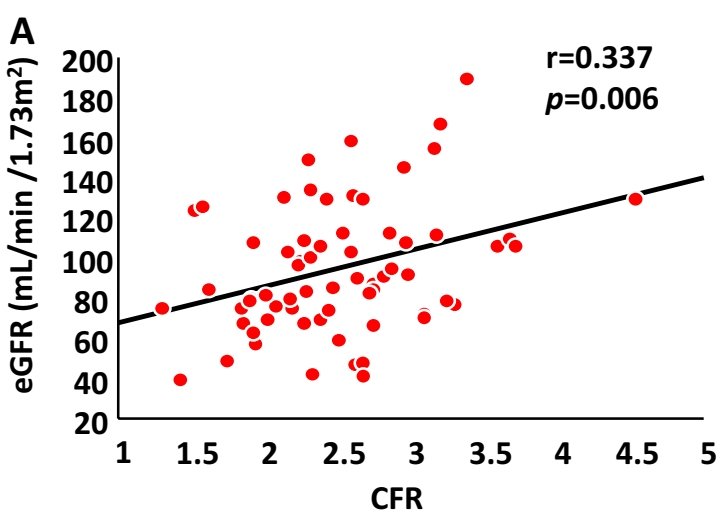

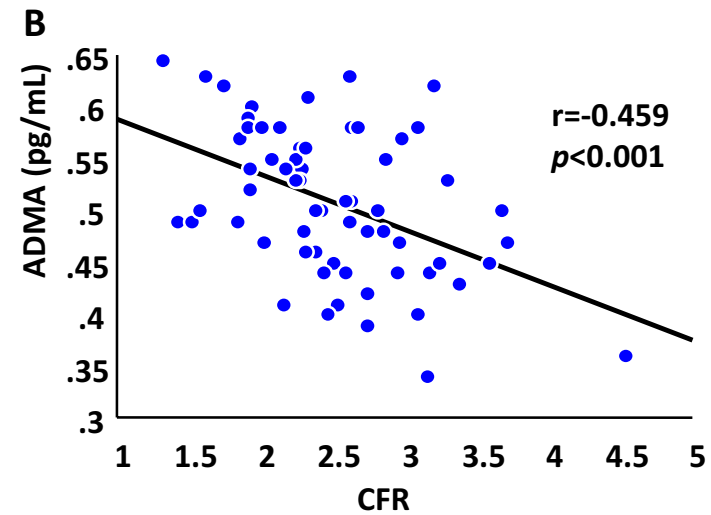

Fig. 3 Relationship among eGFR, CFR, and ADMA. a Relationship between eGFR and CFR. b Relationship between ADMA and CFR. c Relationship between ADMA and eGFR. $e G F R$ estimated glomeru-

factors of vascular calcification [28]. Among the various risk factors, mineral bone disorder is believed to be the most crucial factor for patients with CKD. The underlying mechanisms include the role of elevated serum phosphate levels, parathyroid hormone levels, and fibroblast growth factor 23 levels as well as decreased active vitamin $\mathrm{D}$ and klotho. Although these factors exert a considerable influence on the progression of vascular calcification in CKD, phosphate is the most important factor [28, 35]. The supposed mechanisms of vascular calcification involve the transformation of vascular smooth muscle cells into osteoblast-like cells by the uptake of phosphorus into cells through sodium-dependent phosphorus co-transporters and decrease of inhibitors against vascular calcification [35]. Even in the general population, serum phosphate levels are significantly associated with CAC prevalence [36]. Serum phosphate levels are also significantly associated with not only increased CAD, but also increased the other CVD events $[37,38]$. Furthermore, the results of a meta-analysis have demonstrated that the presence of vascular calcification is significantly associated with higher CVD events and mortality [39].

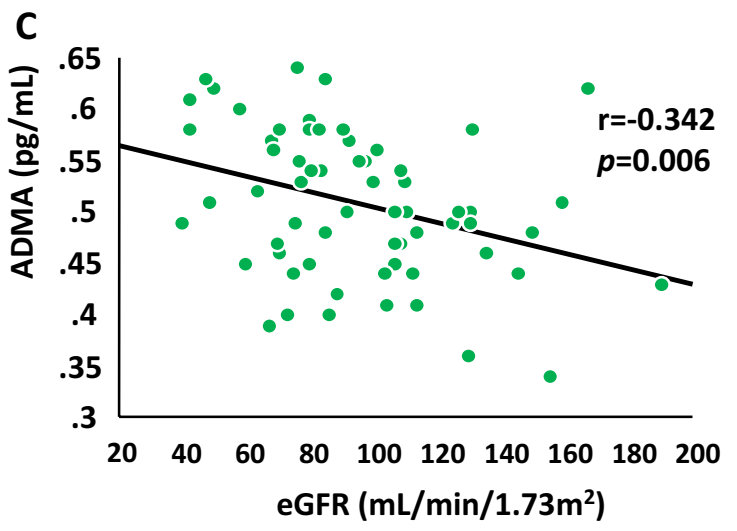

Fujii H, et al. Am J Hypertens. 2008;21:650-656.

lar filtration rate, $C F R$ coronary flow reserve, $A D M A$, asymmetric dimethylarginine

\section{Impact of CAC on CAD in CKD}

The pathophysiology of vascular lesions related to CAD is broadly classified into atherosclerosis and arteriolosclerosis.

American Heart Association has reported the Stary classification for atherosclerosis of coronary artery (Table 1) [40]. As per this classification, advanced types of atheroma, such as type 4 and 5, include calcific lesions. As mentioned above, CAC is a very important and prominent vascular lesion for CKD. There are two types of vascular calcifications based on the mechanisms of vascular calcific formation: one mechanism involves atherosclerotic calcification occurring in the intimal layer of the artery, while the other involves Monckeberg's arteriosclerosis that occurs in the medial layer of the artery. Although these two types of vascular calcifications can be observed in patients with CKD, Monckeberg's arteriosclerosis is often more prominent. With the progression of the stage of CKD, vascular calcification progresses and is a very crucial clinical problem, particularly in patients with CKD stage 5D (those undergoing dialysis).

Acute coronary syndrome (ACS) is a syndrome that includes AMI and unstable angina pectoris that leads to 
Table 1 Stary classification Stary et al. [40]

\begin{tabular}{lll}
\hline Classification & Histology & Main mechanisms of progression \\
\hline Type I (initial lesion) & $\begin{array}{c}\text { Increase in macrophages and formation of scattered mac- } \\
\text { rophage foam cells }\end{array}$ & Lipid accumulation \\
Type II (fatty streak lesion) & Mainly intracellular lipid accumulation & Lipid accumulation \\
Type III (intermediate lesion) & Type II + extracellular lipid pools & Lipid accumulation \\
Type IV (atheroma lesion) & Type II + extracellular lipid core & Lipid accumulation \\
Type V (fibroatheroma lesion) & Lesion with lipid core, fibrous cap, and calcification & Increase of VSMC and a fibrous component \\
Type VI (complicated lesion) & Lesion with ulceration, hemorrhage, and thrombus & Hemorrhage, thrombus, and hematoma \\
\hline
\end{tabular}

VSMC vascular smooth muscle cell

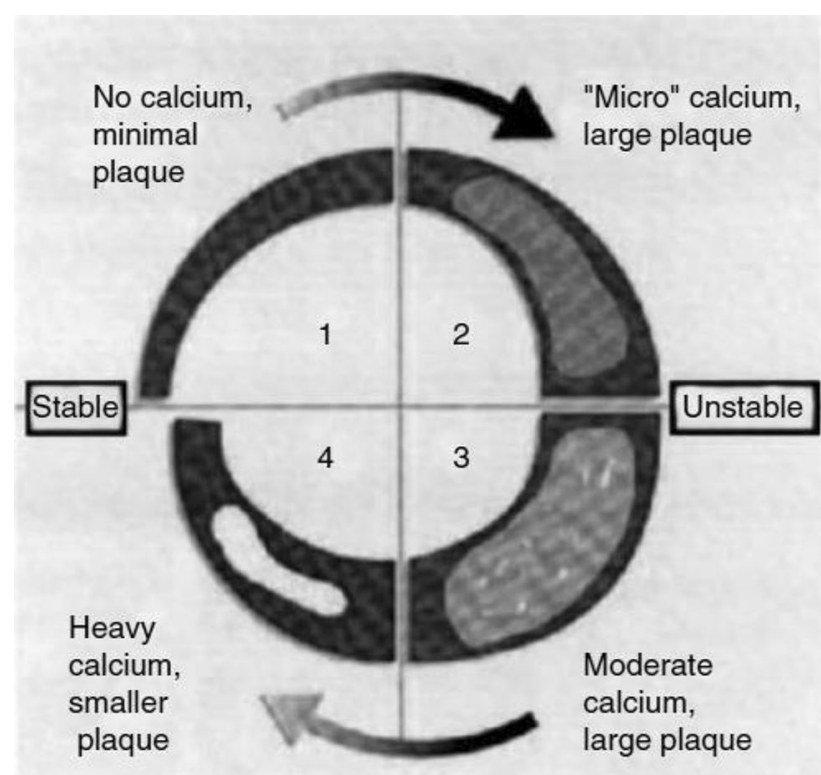

Schoenhagen P. et al. Cleve Clin J Med. 2002;(69) Supple 3:S12-S20.

Fig. 4 Impact of the degree of vascular calcification on plaque stability. Coronary artery calcification alters plaque stability depending on the size and distribution of the calcium deposit. Spotty calcification is strongly associated with the occurrence of acute coronary syndrome

myocardial damage and death due to decreased coronary blood flow. The causes of ACS involve rupture of unstable plaque and thrombus formation in the coronary artery. The stability of plaque is reportedly related to calcification (Fig. 4) [41]. Plaque with trivial or mild calcification is unstable; however, plaque with severe calcification becomes stable. In our previous study that assessed a composition of culprit coronary lesions in patients with CKD demonstrated that the percentage of necrotic core and dense calcium significantly increased in advanced CKD and a calcific lesion was particularly prominent (Fig. 5) [42]. In agreement with the previous reports [43], our study also proved that the necrotic core/dense calcium ratio was significantly higher in patients with ACS than in those with stable angina pectoris [42].
In addition to atherosclerosis, arteriolosclerosis and endothelial dysfunction are also of critical importance in CKD. Not only significant coronary artery stenosis but also arteriolosclerosis and endothelial dysfunction result in impaired coronary microcirculation and lead to cardiac hypertrophy and myocardial fibrosis. CFR reflects severity of arteriolosclerosis and endothelial dysfunction, and CAC proved to be inversely associated with CFR [44-46]. In patients with advanced CKD including those undergoing dialysis, elevations of cardiac biomarkers such as troponin $\mathrm{T}$ and I, patchy myocardial fibrosis, and chronic myocardial injury are often observed [47, 48]. Such myocardial ischemia in advanced CKD is diffuse. Therefore, majority do not show typical ECG changes, such as ST elevation and abnormal $\mathrm{Q}$ wave in myocardial infarction (MI). We speculate that these pathophysiological mechanisms may be the underlying reason for the high incidence of congestive heart failure and type 2 myocardial infarction (non-ST elevation MI without a significant coronary artery stenosis) in advanced CKD.

\section{Treatment of CAD in CKD}

In general, aggressive treatment for CAD involves percutaneous coronary intervention (PCI) and coronary artery bypass grafting (CABG). It is very challenging to decide which treatment is better for patients with CKD, and the strategy is controversial. A randomized-controlled trial (RCT) in patients with severe CAD demonstrated that CABG resulted in lower rates of the combined end point of major adverse cardiac or cerebrovascular events at 1 year compared to PCI [49]. Another RCT conducted on subjects with multivessel CAD and diabetes mellitus also demonstrated that $\mathrm{CABG}$ reduced the mortality rates and MI prevalence significantly more than PCI [50].

In contrast, limited information is available regarding this issue in CKD, because, to our knowledge, no RCT has been conducted on patients with CKD until date. The results of a previous meta-analysis that included patients with CKD except for those undergoing dialysis reported no significant 

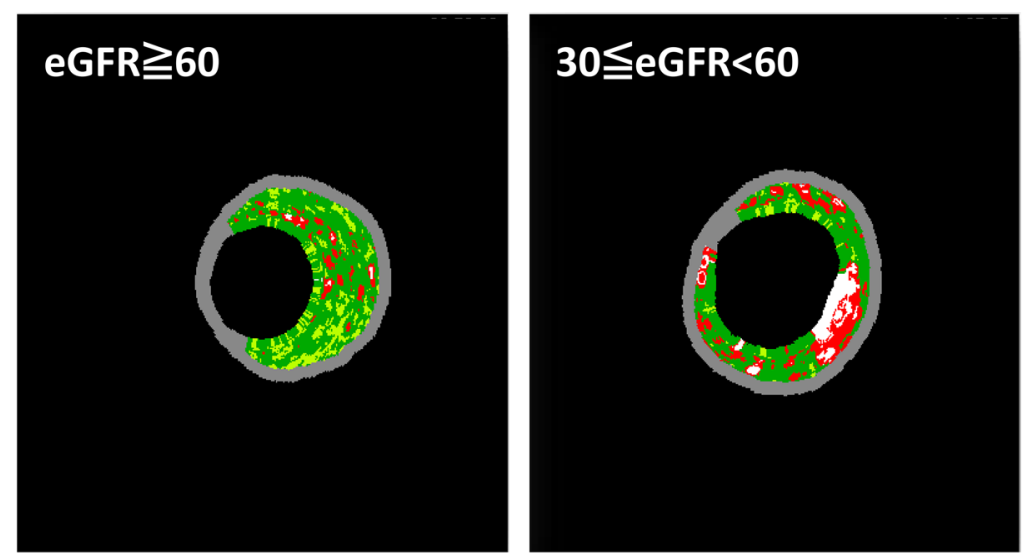

Fibrous
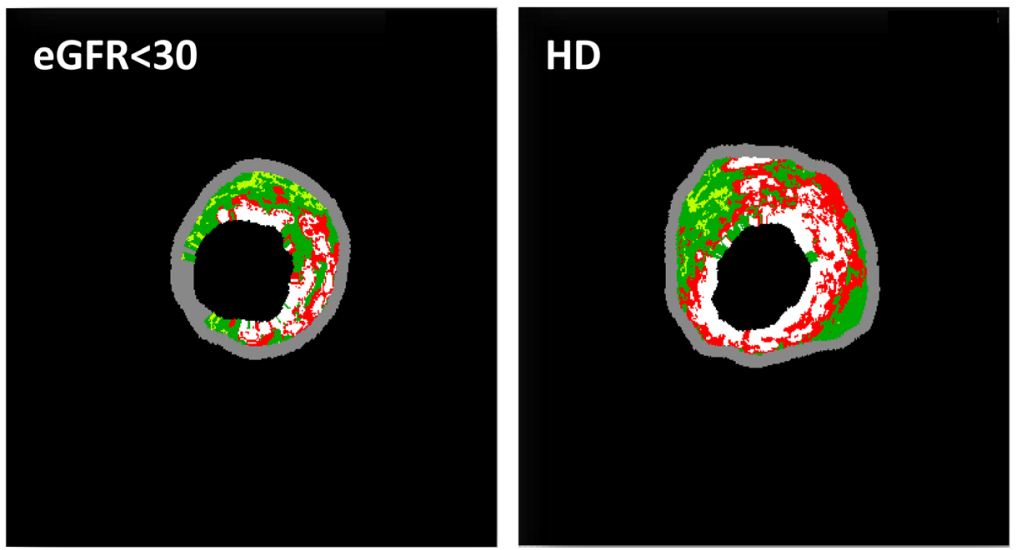

Kono K, et al. Kidney Int. 2012:82;344-351.

Fig. 5 Visualized images of the coronary plaque morphology in each CKD stage. $C K D$ chronic kidney disease

differences in the mortality between the CABG and PCI groups; however, CABG was superior to PCI in terms of the occurrence of $\mathrm{MI}$ and revascularization [51]. With respect to patients undergoing dialysis, a previous observational study using multivariable adjusted proportional hazards regression and a propensity score-matched cohort have revealed that, as compared to PCI, CABG was associated with significantly lower risks for both, death and the composite of death or MI [52]. A meta-analysis has shown that the shortterm mortality is higher and the long-term cardiac event rate is lower in the CABG group as compared to that in the PCI group [53]. Although the meta-analysis also reported no significant differences in the long-term mortality, several critical issues were included in this study. First, these data are mainly from foreign countries other than Japan. Japanese patients undergoing dialysis are reported to have much better prognosis than patients undergoing dialysis in the other countries [54]. If an intervention study that targets patients with CAD undergoing dialysis is performed in Japan, favorable results can be obtained. Second, PCI is a treatment for a local vascular lesion, and CABG is a treatment for the total vessel. Patients undergoing dialysis have severe vascular calcification; therefore, cardiologists commonly encounter coronary lesions for which PCI is challenging in the clinical setting. In severe CAC, stent delivery for a target lesion is often disturbed. Furthermore, patients undergoing dialysis commonly exhibit multivessel lesions. Thus, we believe that CABG should be selected for patients undergoing dialysis if the patient condition is relatively stable.

Taking these findings into account, we propose the following indications of PCI for patients with CKD; (1) an emergency case, (2) early-to-moderate stage CKD, (3) high risk involved in surgical approach, (4) short expected life span, and (5) contraindication for CABG (single-vessel disease or two-vessel disease except for left anterior descending and/or left main trunk).

\section{Conclusion}

The course of CAD in patients with CKD is different from that in those without CKD. Thus, occasionally, common protocol followed for patients without CKD may not be applicable to those with CKD. Furthermore, the available data 
regarding CKD have been derived from clinical studies that did not involve patients with CKD. Therefore, these data may not applicable to patients with CKD. Thus, the treatment decision for patients with CKD should be made after considering the patient's clinical characteristics.

Acknowledgements I would like to greatly thank the Japanese Society of Nephrology for this award.

\section{Compliance with ethical standards}

Conflict of interest H.F. received lecture fees from Chugai Pharmaceutical Co., Ltd., Bayer Yakuhin Co., Ltd., KISSEI Pharmaceutical Co., Ltd., and ONO Pharmaceutical Co., Ltd., and scholarship/donation from Chugai Pharmaceutical Co., Ltd., Bayer Yakuhin Co., Ltd. and ONO Pharmaceutical Co., Ltd., MSD, Asteras, TEIJIN and KYOWA HAKKO KIRIN Pharmaceutical Co., Ltd. S.N. received lecture fees from Chugai Pharmaceutical Co., Ltd., Bayer Yakuhin Co., Ltd., KYOWA HAKKO KIRIN Pharmaceutical Co., and Asteras Pharmaceutical Co., Ltd., and scholarship/donation from Chugai Pharmaceutical Co., Ltd., Bayer Yakuhin Co., Ltd., and ONO Pharmaceutical Co., Ltd., MSD, Asteras, TEIJIN and KYOWA HAKKO KIRIN Pharmaceutical Co., Ltd. All other authors declared no other conflict of interest.

Human and animal rights Not applicable.

Informed consent Not applicable. Contents of this manuscript were partly presented as the Clinical Scientist Award memorial lecture at the 61th annual meeting of the Japanese Society of Nephrology, 2018.

Open Access This article is distributed under the terms of the Creative Commons Attribution 4.0 International License (http://creativeco mmons.org/licenses/by/4.0/), which permits unrestricted use, distribution, and reproduction in any medium, provided you give appropriate credit to the original author(s) and the source, provide a link to the Creative Commons license, and indicate if changes were made.

\section{References}

1. Ronco C, Cicoira M, McCullough PA. Cardiorenal syndrome type 1: pathophysiological crosstalk leading to combined heart and kidney dysfunction in the setting of acutely decompensated heart failure. J Am Coll Cardiol. 2012;60:1031-42.

2. Sarnak MJ, Levey AS, Schoolwerth AC, et al. Kidney disease as a risk factor for development of cardiovascular disease: a statement from the American Heart Association Councils on Kidney in Cardiovascular Disease, High Blood Pressure Research, Clinical Cardiology, and Epidemiology and Prevention. Circulation. 2003; 108:2154-69.

3. Bae EH, Lim SY, Cho KH, et al. GFR and cardiovascular outcomes after acute myocardial infarction: results from the Korea Acute Myocardial Infarction Registry. Am J Kidney Dis. 2012;59(6):795-802.

4. Nakano T, Ninomiya $T$, Sumiyoshi $S$, et al. Association of kidney function with coronary atherosclerosis and calcification in autopsy samples from Japanese elders: the Hisayama study. Am J Kidney Dis. 2010;55(1):21-30.
5. Anaveker NS, McMurray JJ, Velazquez EJ, et al. Relation between renal dysfunction and cardiovascular outcomes after myocardial infarction. N Engl J Med 2004;1285-1295.

6. Tonelli M, Muntner P, Lloyd A, et al. Risk of coronary events in people with chronic kidney disease compared with those with diabetes: a population-level cohort study. Lancet. 2012;380(9844):807-14.

7. Masakane I, Nakai S, Ogata S, et al. An overview of regular dialysis treatment in Japan (As of 31 December 2013). Ther Apher Dial. 2015;19(6):540-74.

8. Shroff GR, Herzog CA. Acute myocardial infarction in patients with chronic kidney disease: how are the most vulnerable patients doing? Kidney Int. 2013:84;230-233.

9. Trespalacios FC, Taylor AJ, Agodoa LY. Incident acute coronary syndromes in chronic dialysis patients in the United States. Kidney Int. 2002:62;1799-1805.

10. Joki N, Hase H, Nakamura R, et al. Onset of coronary artery disease prior to initiation of haemodialysis in patients with end-stage renal disease. Nephrol Dial Transplant. 1997;12(4):718-23.

11. Ohtake T, Kobayashi S, Moriya H, et al. High prevalence of occult coronary artery stenosis in patients with chronic kidney disease at the initiation of renal replacement therapy: an angiographic examination. J Am Soc Nephrol. 2005;16:1141-8.

12. Iwasaki M, Joki N, Tanaka Y, et al. Declining prevalence of coronary artery disease in incident dialysis patients over the past 2 decades. J Atheroscler Thromb. 2016;23:491.

13. Herzog CA, Littrell K, Arko C, et al. Clinical characteristics of dialysis patients with acute myocardial infarction in the United States: a collaborative project of the United States Renal Data System and the National Registry of Myocardial Infarction. Circulation. 2007;116:1465-72.

14. Perticone F, Ceravolo R, Pujia A, et al. Prognostic significance of endothelial dysfunction in hypertensive patients. Circulation. 2001;104:191-6.

15. Go AS, Chertow GM, Fan D, et al. Chronic kidney disease and the risks of death, cardiovascular events, and hospitalization. $\mathrm{N}$ Engl J Med. 2004;351:1296-305.

16. Gerstein HC, Mann JF, Yi Q, et al. HOPE Study Investigators. Albuminuria and risk of cardiovascular events, death, and heart failure in diabetic and nondiabetic individuals. JAMA. 2001;286:421-6.

17. Opherk D, Mall G, Zebe H, et al. Reduction of coronary reserve: a mechanism for angina pectoris in patients with arterial hypertension and normal coronary arteries. Circulation. 1984;69:1-7.

18. Kern MJ, Lerman A, Bech JW, et al. Physiological assessment of coronary artery disease in the cardiac catheterization laboratory: a scientific statement from the American Heart Association Committee on Diagnostic and Interventional Cardiac Catheterization, Council on Clinical Cardiology. Circulation. 2006;114:1321-41.

19. Chade AR, Brosh D, Higano ST, et al. Mild renal insufficiency is associated with reduced coronary flow in patients with nonobstructive coronary artery disease. Kidney Int. 2006;69:266-71.

20. Fujii H, Takiuchi S, Kawano Y, et al. Putative role of asymmetric dimethylarginine in microvascular disease of kidney and heart in hypertensive patients. Am J Hypertens. 2008;21:650-6.

21. Mohandas R, Segal MS, Huo T, et al. Renal function and coronary microvascular dysfunction in women with symptoms/signs of ischemia. PLoS One. 2015;10:e0125374.

22. Takiuchi S, Fujii H, Kamide K, et al. Plasma asymmetric dimethylarginine and coronary and peripheral endothelial dysfunction in hypertensive patients. Am J Hypertens. 2004;17(9):802-8.

23. Fujii H, Takiuchi S, Kamide K, et al. Clinical implications of assessing coronary flow velocity reserve and plasma endothelin- 1 in hypertensive patients. Hypertens Res. 2005;28(11):911-6. 
24. Fujii H, Kono K, Yamamoto T, et al. Effect of enzyme replacement therapy on serum asymmetric dimethylarginine levels, coronary flow reserve and left ventricular hypertrophy in patients with fabry disease. Clin Kidney J. 2012;5:512-8.

25. Charytan DM, Skali H, Shah NR, et al. Coronary flow reserve is predictive of the risk of cardiovascular death regardless of chronic kidney disease stage. Kidney Int. 2018;93:501-9.

26. Shar NR, Charytan DM, Murthy VL, et al. Prognostic value of coronary flow reserve in patients with dialysis-dependent ESRD. J Am Soc Nephrol. 2016;27:1823-9.

27. Stabley JN, Towler DA. Arterial calcification in diabetes mellitus: preclinical models and translational implications. Arterioscler Thromb Vasc Biol. 2017;37:205-17.

28. Vervloet M, Cozzolino M. Vascular calcification in chronic kidney disease: different bricks in the wall? Kidney Int. 2017;91(4):808-17.

29. Fernández-Friera L, Peñalvo JL, Fernández-Ortiz A, et al. Prevalence, vascular distribution, and multiterritorial extent of subclinical atherosclerosis in a middle-aged cohort: the PESA (Progression of Early Subclinical Atherosclerosis) Study. Circulation. 2015;131:2104-13.

30. Kaplan H, Thompson RC, Trumble BC, et al. Coronary atherosclerosis in indigenous South American Tsimane: a cross-sectional cohort study. Lancet. 2017;389(10080):1730-9.

31. Watanabe R, Lemos MM, Manfredi SR, et al. Impact of cardiovascular calcification in nondialyzed patients after 24 months of follow-up. Clin J Soc Am Nephrol. 2010:5;189-194.

32. Kitamura K, Fujii H, Kono K, et al. Relationship between cardiac calcification and left ventricular hypertrophy in patients with chronic kidney disease at hemodialysis initiation. Heart Vessels. 2017;32(9):1109-16.

33. Detrano R, Guerci AD, Carr JJ, et al. Coronary calcium as a predictor of coronary events in four racial or ethnic groups. N Engl J Med. 2008;358:1336-45.

34. Chiu YW, Adler SG, Budoff MJ, et al. Coronary artery calcification and mortality in diabetic patients with proteinuria. Kidney Int. 2010;77:1107-14.

35. Jono $\mathrm{S}$, McKee MD, Murry $\mathrm{CE}$, et al. Phosphate regulation of vascular smooth muscle cell calcification. Circ Res. 2000;87(7):E10-7.

36. Foley RN, Collins AJ, Herzog CA, et al. Serum phosphorus levels associate with coronary atherosclerosis in young adults. J Am Soc Nephrol. 2009;20:397-404.

37. Dhingra R, Sullivan LM, Fox CS, et al. Relations of serum phosphorus and calcium levels to the incidence of cardiovascular disease in the community. Arch Intern Med. 2007;167:879-85.

38. Tonelli M, Sacks F, Pfeffer M, et al. Relation between serum phosphate level and cardiovascular event rate in people with coronary disease. Circulation. 2005;112:2627-33.

39. Rennenberg RJ, Kessels AG, Schurgers LJ, et al. Vascular calcifications as a marker of increased cardiovascular risk: a metaanalysis. Vasc Health Risk Manag. 2009;5:185-97.

40. Stary HC, Chandler AB, Dinsmore RE, et al. A definition of advanced types of atherosclerotic lesions and a histological classification of atherosclerosis. A report from the Committee on
Vascular Lesions of the Council on Arteriosclerosis, American Heart Association. Circulation. 1995;92:1355-74.

41. Schoenhagen P, Tuzcu EM. Coronary artery calcification and endstage renal disease: vascular biology and clinical implications. Clevel Clin J Med. 2002;69(Suppl 3):12-20.

42. Kono K, Fujii H, Nakai K, et al. Composition and plaque patterns of coronary culprit lesions and clinical characteristics of patients with chronic kidney disease. Kidney Int. 2012;82:344-51.

43. Ehara S, Kobayashi Y, Yoshiyama M, et al. Spotty calcification typifies the culprit plaque in patients with acute myocardial infarction: an intravascular ultrasound study. Circulation. 2004;110:3424-9.

44. Kim J, Bravo PE, Gholamrezanezhad A, et al. Coronary artery and thoracic aorta calcification is inversely related to coronary flow reserve as measured by ${ }^{82} \mathrm{Rb}$ PET/CT in intermediate risk patients. J Nucl Cardiol. 2013;20(3):375-84.

45. Wang L, Jerosch-Herold M, Jacobs DR Jr, et al. Coronary artery calcification and myocardial perfusion in asymptomatic adults: the MESA (Multi-Ethnic Study of Atherosclerosis). J Am Coll Cardiol. 2006;48(5):1018-26.

46. Caliskan Y, Demirturk M, Ozkok A, et al. Coronary artery calcification and coronary flow velocity in haemodialysis patients. Nephrol Dial Transplant. 2010;25(8):2685-90.

47. Khan NA, Hemmelgarn BR, Tonelli M, et al. Prognostic value of troponin $\mathrm{T}$ and I among asymptomatic patients with end-stage renal disease: a meta-analysis. Circulation. 2005;112:3088-96.

48. Tonelli M, Karumanchi SA, Thadhani R. Epidemiology and mechanisms of uremia-related cardiovascular disease. Circulation. 2016;133:518-36.

49. Serruys PW, Morice MC, Kappetein AP, et al. Percutaneous coronary intervention versus coronary-artery bypass grafting for severe coronary artery disease. N Engl J Med. 2009;360:961-72.

50. Farkouh ME, Domanski M, Sleeper LA, et al. Strategies for multivessel revascularization in patients with diabetes. N Engl J Med. 2012; 367: 2375-2384.

51. Charytan DM, Desai M, Mathur M, et al. Reduced risk of myocardial infarct and revascularization following coronary artery bypass grafting compared with percutaneous coronary intervention in patients with chronic kidney disease. Kidney Int. 2016;90:411-21.

52. Chang TI, Shilane D, Kazi DS, et al. Multivessel coronary artery bypass grafting versus percutaneous coronary intervention in ESRD. J Am Soc Nephrol. 2012;23:2042-9.

53. Nevis IF, Mathew A, Novick RJ, et al. Optimal method of coronary revascularization in patients receiving dialysis: systematic review. Clin J Am Soc Nephrol. 2009;4(2):369-78.

54. Yoshino M, Kuhlmann MK, Kotanko P, et al. International differences in dialysis mortality reflect background general population atherosclerotic cardiovascular mortality. J Am Soc Nephrol. 2006;17(12):3510-9.

Publisher's Note Springer Nature remains neutral with regard to jurisdictional claims in published maps and institutional affiliations. 